FUB-HEP 9/92

HLRZ Preprint 56/92

August 1992

\title{
Finite-Size Scaling Study of the Three-Dimensional Classical Heisenberg Model
}

\author{
Christian Holm ${ }^{1}$ and Wolfhard Janke ${ }^{2}$ \\ 1 Institut für Theoretische Physik, Freie Universität Berlin \\ Arnimallee 14, D-1000 Berlin 33, Germany \\ 2 Höchstleistungsrechenzentrum, Forschungszentrum Jülich \\ Postfach 1913, D-5170 Jülich, Germany
}

\begin{abstract}
We use the single-cluster Monte Carlo update algorithm to simulate the three-dimensional classical Heisenberg model in the critical region on simple cubic lattices of size $L^{3}$ with $L=12,16,20,24,32,40$, and 48. By means of finite-size scaling analyses we compute high-precision estimates of the critical temperature and the critical exponents, using extensively histogram reweighting and optimization techniques. Measurements of the autocorrelation time show the expected reduction of critical slowing down at the phase transition. This allows simulations on significantly larger lattices than in previous studies and consequently a better control over systematic errors in finite-size scaling analyses.
\end{abstract}

*Work supported in part by Deutsche Forschungsgemeinschaft under grant Kl256. 
The critical behaviour of the three-dimensional (3D) classical Heisenberg model, as one of the simplest spin models, has been investigated by a variety of approaches. Despite this fact there are still some discrepancies to be resolved. Motivated by conflicting estimates of its critical coupling $\beta_{c}$ on a simple cubic lattice coming from widely accepted high-temperature series expansion analyses [四 $\left(\beta_{c} \approx 0.6916\right)$ and more recent transfer-matrix (TM) Monte Carlo (MC) investigations [2] $\left(\beta_{c}=0.6922(1)\right.$ and $\left.\beta_{c}=0.6925(3)\right)$, Peczak et al. [3] (PFL) have recently undertaken a high statistics MC study of this model on cubic lattices of sizes up to $V=L^{3}=24^{3}$. By simulating the system with the standard Metropolis algorithm [4] and making extensive use of multi-histogram techniques [5], they could not decide between the two alternatives. Rather, from a finite-size scaling (FSS) [6] analysis of the crossing points of Binder's cumulant [0] they claimed an even larger value of $\beta_{c}=0.6929(1)$. Moreover, extrapolations of the locations of the susceptibility and specific-heat peak maximum to the infinite volume limit yielded [3], respectively, $\beta_{c}=0.6930(2)$ and $\beta_{c}=0.6931(10)$, in agreement with the cumulant crossing value.

However, PFL did not mention a later reanalysis [8] of the high-temperature series expansion based on the Padé $\left(\beta_{c}=0.6924(2)\right)$ and ratio $\left(\beta_{c}=0.6925(1)\right)$ method, respectively. While these values are consistent with the TM estimates given in ref.[2], we are now faced with the problem that the latest MC result is significantly higher.

The critical coupling is a non-universal parameter and from this point of view not of particular interest. Most estimates of universal critical indices, however, are biased and usually depend quite strongly on the precise value of $\beta_{c}$. To clarify the above discrepancy we found it therefore worthwhile to perform an independent high precision MC study on larger lattices of sizes up to $48^{3}$ and with even higher statistics as in PFL's work. Relying on the Metropolis algorithm as PFL did, such a project would have been hardly feasible. As with most local algorithms, the Metropolis (pseudo) dynamics suffers from the severe problem of critical slowing down, that is from large autocorrelation times $\tau=a L^{z}$ (with dynamical critical exponent $z=1.94(6)$ (ref. [9]) and $a \approx 3.76$ ), which reduce the size of the statistical sample, $N$, effectively to $N_{\text {eff }}=N / 2 \tau$. It is therefore crucial to use one of the improved algorithms [10] of the past few years that avoid this problem. We chose the

\footnotetext{
${ }^{1}$ This can be read off from Fig. 2 in ref. [9].
} 
cluster algorithm [11] in its single-cluster variant [12]. From studies of related spin models it is known [13] that this update algorithm is extremely efficient in three dimensions.

The classical Heisenberg model is defined by the partition function

$$
Z=\prod_{i}\left[\int \frac{d \phi_{i} d \cos \theta_{i}}{4 \pi}\right] e^{-\beta E},
$$

where $\beta \equiv 1 / T$ is the (reduced) inverse temperature and

$$
E=\sum_{\langle i, j\rangle}\left[1-\vec{s}_{i} \cdot \vec{s}_{j}\right]
$$

is the total energy (in the following small letters always denote the corresponding intensive quantities, e.g., $e \equiv E / V)$. The sum runs over all nearest neighbour pairs $\langle i, j\rangle$ and the three-dimensional unit spins $\vec{s}$ at the sites $i$ of a simple cubic lattice are parametrized as $\vec{s}=(\cos \phi \sin \theta, \sin \phi \sin \theta, \cos \theta)$. We always employ periodic boundary conditions.

Our simulations were organized as follows. First, we did one run for each lattice size at $\beta_{0}=0.6929$, the estimate of $\beta_{c}$ by PFL, and recorded the energy histogram $P_{\beta_{0}}(E)$ and the microcanonical averages $\left\langle\left\langle m^{k}\right\rangle\right\rangle(E) \equiv$ $\sum_{M} P_{\beta_{0}}(E, M) m^{k} / P_{\beta_{0}}(E), k=1,2,4$, where $m=|\vec{m}|$ is the magnitude of the magnetization $\vec{m}=\frac{1}{V} \sum \boldsymbol{x} \vec{s}(\boldsymbol{x})$ of a single spin configuration. The temperature independent averages $\left\langle\left\langle m^{k}\right\rangle\right\rangle(E)$ can be computed by accumulating the values of $m^{k}$ in lists indexed by the associated energy bin of the configuration and normalizing at the end by the total number of entries in each bin, making it thus unnecessary to store the two-dimensional histogram $P_{\beta_{0}}(E, M)$. The continuous energy range $0 \leq E \leq 3 \mathrm{~V}$ was discretized into 90000 bins. The data of this run is sufficient to compute the approximate positions $\beta_{-}<\beta_{0}$ and $\beta_{+}>\beta_{0}$ of the (connected) susceptibility and the specific-heat peak maximum by reweighting techniques [5]. We then performed two more runs at $\beta_{-}$and $\beta_{+}$, respectively, again recording $P_{\beta}(E)$ and $\left\langle\left\langle m^{k}\right\rangle\right\rangle(E)$. This choice has the advantage that one automatically stays in the critical region since both $\beta_{-}$and $\beta_{+}$scale with $L^{-1 / \nu}$, where $\nu$ is the correlation length exponent. From this data we can compute three estimates $\mathcal{O}_{L}^{(n)}(\beta), n=-, 0,+$ for all thermodynamic observables $\mathcal{O}_{L}$ of interest, and for any $\beta$ value in the vicinity of $\beta_{-}, \beta_{0}, \beta_{+}$by reweighting . Furthermore, since we devided the whole run into several blocks and stored the energy histograms and microcanonical averages for each block, we could compute jackknife errors [14] $\Delta \mathcal{O}_{L}^{(n)}$ 
on $\mathcal{O}_{L}^{(n)}$. This allowed us to get an optimized average of these three values that minimizes the relative error of the combined $\mathcal{O}_{L}(\beta)$ for each observable separately (the relative weights are simply $\left.1 /\left(\Delta \mathcal{O}_{L}^{(n)}\right)^{2}\right)$. All our runs contain at least $10000 \times \tau$ measurements, where $\tau$ is the integrated autocorrelation time of the susceptibility. As expected for the single-cluster update, $\tau$ turns out to be almost independent of the lattice size and to be very small $(<2$, in units of lattice sweeps that allow direct comparison with the Metropolis algorithm). For the $48^{3}$ lattice, $\tau$ is about three orders of magnitude smaller than for the Metropolis algorithm. This explains why we could study much larger lattice sizes than PFL, and could still afford to have about ten times better statistics.

To determine $\beta_{c}$ we first concentrated on Binder's cumulant [7]

$$
U_{L}(\beta)=1-\frac{\left\langle m^{4}\right\rangle}{3\left\langle m^{2}\right\rangle^{2}},
$$

where the angular brackets $\langle\ldots\rangle$ denote thermal averages with respect to (1). It is well known [7] that, asymptotically for large $L$, all curves $U_{L}(\beta)$ should cross in the unique point $\left(\beta_{c}, U^{*}\right)$. Our results for lattices of size $L=12,16$, $20,24,32,40$, and 48 , obtained by the optimization procedure described above, are shown in Fig. 1. We see that all curves indeed cross each other at approximately the same $\beta$ value of 0.693 . A closer look, however, reveals that the crossing points of $U_{L}$ and $U_{L^{\prime}}$ are systematically shifted, depending on the ratio $b \equiv L / L^{\prime}$. This is the expected behaviour for finite lattices, caused by confluent correction terms. Employing well-known [7] extrapolation formulas we obtain the final estimates 15

$$
\beta_{c}=0.6930 \pm 0.0001,
$$

and

$$
U^{*}=0.6217 \pm 0.0008 .
$$

The critical coupling is thus found in excellent agreement with the value quoted by PFL, and also $U^{*}$ agrees very well with their estimate of $0.622(1)$. For comparison, a field theoretic expansion in $\sqrt{4-D}$ predicts for $D=3$ a $4 \%$ lower value of $0.59684 \ldots$ [16.

Let us now turn to the FSS estimates of critical exponents. The derivatives $d U_{L} / d \beta$ at $\beta_{c}=0.6930$ should scale asymptotically for large $L$ with 
$L^{1 / \nu}$. In a log-log plot of all our data points we find a perfect straight line fit (with goodness-of-fit parameter $Q=0.61$ ) and from the inverse slope we read off

$$
\nu=0.704 \pm 0.006,
$$

which again is in agreement with the value quoted by PFL, $\nu=0.706(9)$ (determined by the same method, but at $\beta=0.6929$ ), and with the field theoretical estimates of $\nu=0.705(3)$ (resummed perturbation expansion [17]), $\nu=0.710(7)$ (resummed $\epsilon$-expansion [18]). The high quality of this fit (as well as of all other fits described below) shows that the asymptotic scaling formula works down to our smallest lattice size $L=12$, indicating that there is no need for confluent correction terms.

The ratio of exponents $\beta / \nu$ follows from the scaling of the magnetization, $\langle m\rangle \propto L^{-\beta / \nu}$. In a $\log -\log$ plot of $\langle m\rangle$ at $\beta_{c}=0.6930$ vs $L$ we obtain from a straight line fit (with $Q=0.68$ ) the estimate

$$
\beta / \nu=0.514 \pm 0.001,
$$

which is slightly lower than the value given by PFL, $\beta / \nu=0.516$ (3) (determined at $\beta=0.6929)$. To test by how much our result is biased by the value of $\beta_{c}$ we have redone our analysis at $\beta=0.6929$. Here we get the slightly higher value of $0.519(1)$. The quality of the fit, however, is much worse $(Q=0.30)$. Since we observe a similar worsening of the fit at $\beta=0.6931$ $(\beta / \nu=0.509(1), Q=0.31)$, we take this as support for our estimate of $\beta_{c}$. We rely on the goodness-of-fit parameter since visually it is impossible to make a distinction between these fits when plotted on a natural scale. It should be emphasized that even these slight variations in the estimate of the critical coupling produce significant changes in the estimate of the exponent ratio that clearly dominate the statistical errors. Combining (66) and (7) we get for the critical exponent $\beta=0.362(4)$, to be compared with the estimates of $\beta=0.3645(25)$ (ref. 17]) and $\beta=0.368(4)$ (ref. 18]).

All other critical exponents follow from (hyper-) scaling relations. In this way we obtain

$$
\begin{gathered}
\alpha / \nu=2 / \nu-D=-0.159 \pm 0.025 \\
\gamma / \nu=3-2 \beta / \nu=1.972 \pm 0.002
\end{gathered}
$$

and

$$
\eta=2-\gamma / \nu=2 \beta / \nu-1=0.028 \pm 0.002 .
$$


To test these relations we have performed also a direct analysis of the critical behaviour of the specific heat

$$
C=V \beta^{2}\left(\left\langle e^{2}\right\rangle-\langle e\rangle^{2}\right) \propto L^{\alpha / \nu},
$$

and of the (connected) susceptibility

$$
\chi^{\mathrm{c}}=V \beta\left(\left\langle m^{2}\right\rangle-\langle m\rangle^{2}\right) \propto L^{\gamma / \nu} .
$$

Since the magnetization vanishes at and above the critical temperature we may also take

$$
\chi=V \beta\left\langle m^{2}\right\rangle \propto L^{\gamma / \nu},
$$

which is usually a less noisy estimator.

From the location of the maxima of $C$ and $\chi^{\mathrm{c}}$ we can get further estimates for the critical coupling by assuming the FSS relation $T_{\max }=T_{c}+a L^{-1 / \nu}+\ldots$. Using our value of $\nu=0.704$ we obtain from the linear fits shown in Fig. 2 the estimates $\beta_{c}=0.6925(9)$ (from $T_{C_{\max }}$ with $Q=0.80$ ) and $\beta_{c}=0.6930(3)$ (from $T_{\chi_{\max }^{\mathrm{c}}}$ with $Q=1.0$ ), respectively. These values are consistent with the cumulant crossing value (雨), but have larger statistical errors.

From the scaling of $\chi^{\mathrm{c}}$ and $\chi$ at our best estimate of $\beta_{c}=0.6930$ we get from linear fits $\eta=0.0156(44)(Q=0.69)$ and $\eta=0.0271(17)(Q=0.78)$, respectively. The latter value is in perfect agreement with the scaling prediction (10). Moreover, performing fits to $\chi$ at $\beta=0.6929$ and $\beta=0.6931$ we obtain estimates of $\eta=0.0364(17)(Q=0.36)$ and $\eta=0.01781(17)$ $(Q=0.43)$, respectively, that are again in perfect agreement with the scaling predictions based on the corresponding fits to the magnetization. This is not unexpected since the measurements of $\langle m\rangle$ and $\chi$ are of course strongly correlated. Finally, analyzing the FSS behaviour of the susceptibility maximum, $\chi_{\max }^{\mathrm{c}} \propto L^{\gamma / \nu}$, we estimate $\eta=0.0231(61)(Q=0.60)$. Notice that all $\mathrm{MC}$ estimates are lower than the field theory values which are $\eta=0.033(4)$ (ref. [17]) and $\eta=0.040(3)$ (ref. [18]) )

The specific heat exhibits a finite, cusp-like singularity, because $\alpha$ has a negative value. We tried a three-parameter fit of the form $C_{\max }=a-$

\footnotetext{
${ }^{2}$ In a recent recalculation [19] of all Feynman graphs several errors in the highest order of the $\epsilon$-expansion were corrected. A subsequent reanalysis [20] of the resummed series gave a slightly smaller value for $\eta$. Compared with the error bar, however, this correction is negligible.
} 
$b L^{\alpha / \nu}$. The result $\alpha / \nu=-0.33(22)(Q=0.69)$ is compatible with the scaling relation (8) but, due to its very large statistical error, it does not provide a stringent test of this scaling prediction. Another way of testing eq. (8) is to assume the predicted value of $\alpha / \nu=-0.159$ and to fit only the parameters $a$ and $b$. The resulting fit turned out to be of almost equally good quality [15].

In summary, using high-precision data from single-cluster $\mathrm{MC}$ simulations combined with optimized multi-histogram techniques, we have performed a fairly detailed FSS analysis of the 3D classical Heisenberg model on simple cubic lattices of size up to $48^{3}$. Qualitatively, our main result is that the asymptotic FSS region sets in for small lattices sizes, $L \approx 12$. Quantitatively, our value for the critical coupling, $\beta_{c}=0.6930(1)$, is in almost perfect agreement with the MC estimate reported recently by PFL (ref. [3]), but is significantly higher than estimates from high-temperature series expansion analyses and transfer matrix methods. Our results for the two basic critical exponents, $\nu=0.704(6)$ and $\beta=0.362(4)$, are in good agreement with field theoretic predictions. Scaling relations imply $\alpha=-0.112(18)$ and $\eta=2-\gamma / \nu=0.028(2)$. Direct measurements of these exponents provide tests of the scaling relations. In the case of $\eta$ we find good agreement when the scaling of $\chi$ at $\beta_{c}$ is considered. Using $\chi^{\mathrm{c}}$ at $\beta_{c}$ or $\chi_{\max }^{\mathrm{c}}$, however, the situation is less clear. In the case of $\alpha$, its negative value causes numerical problems, since a finite, cusp-like singularity is notoriously difficult to analyze.

\section{Acknowledgement}

The numerical simulations were performed on the CRAY X-MP and Y-MP of the Konrad-Zuse Zentrum für Informationstechnik Berlin (ZIB) and the CRAY X-MP at the Rechenzentrum der Universität Kiel. We thank both institutions for their generous support. 


\section{References}

[1] P.J. Wood and G.S. Rushbrooke, Phys. Rev. Lett. 17, 307 (1966);

D.S. Ritchie and M.E. Fisher, Phys. Rev. B5, 2668 (1972).

[2] M.P. Nightingale and H.W.J. Blöte, Phys. Rev. Lett. 60, 1562 (1988).

[3] P. Peczak, A.L. Ferrenberg, and D.P. Landau, Phys. Rev. B43, 6087 (1991).

[4] N. Metropolis, A. W. Rosenbluth, M. N. Rosenbluth, A. H. Teller, and E. Teller, J. Chem. Phys. 21, 1087 (1953).

[5] A.M. Ferrenberg and R.H. Swendsen, Phys. Rev. Lett. 61, 2635 (1988); ibid. 63, 1195 (1989); and Erratum, ibid. 63, 1658 (1989).

[6] M.E. Barber, in Phase Transitions and Critical Phenomena, edited by C.Domb and J.L. Lebowitz (Academic, N.Y., 1983), Vol. 8.

See also the articles in Finite-Size Scaling and Numerical Simulations of Statistical Systems, edited by V. Privman (World Scientific, Singapore, 1990).

[7] K. Binder, Z. Phys. B43, 119 (1981).

[8] S. McKenzie, C. Domb, and D.L. Hunter, J. Phys. A15, 3899 (1982).

[9] P. Peczak and D.P. Landau, J. Appl. Phys. 67, 5427 (1990).

[10] For reviews see, e.g., R.H. Swendsen, J.-S. Wang, and A.M. Ferrenberg, New Monte Carlo Methods for Improved Efficiency of Computer Simulations in Statistical Mechanics, in The Monte Carlo Method in Condensed Matter Physics, edited by K. Binder (Springer, Berlin, 1991);

C.F. Baillie, Int. J. Mod. Phys. C1, 91 (1990);

A.D. Sokal, Monte Carlo Methods in Statistical Mechanics: Foundations and New Algorithms, Cours de Troisième Cycle de la Physique en Suisse Romande, Lausanne, 1989.

[11] R.H. Swendsen and J.-S. Wang, Phys. Rev. Lett. 58, 86 (1987);

J.-S. Wang and R.H. Swendsen, Physica A167, 565 (1990). 
[12] U. Wolff, Phys. Rev. Lett. 62, 361 (1989).

[13] U. Wolff, Phys. Lett. B228, 379 (1989);

J.-S. Wang, Physica A164, 240 (1990);

M. Hasenbusch and S. Meyer, Phys. Lett. B241, 238 (1990);

W. Janke, Phys. Lett. A148, 306 (1990).

[14] R.G. Miller, Biometrica 61, 1 (1974);

B. Efron, The Jackknife, the Bootstrap and other Resampling Plans (SIAM, Philadelphia, PA, 1982).

[15] For more details, see C. Holm and W. Janke, in preparation.

[16] E. Brezin and J. Zinn-Justin, Nucl. Phys. B257[FS14], 867 (1985).

[17] J.C. Le Guillou and J. Zinn-Justin, Phys. Rev. B21, 3976 (1980).

[18] J.C. Le Guillou and J. Zinn-Justin, J. Physique Lett. 46, L137 (1985).

[19] H. Kleinert, J. Neu, V. Schulte-Frohlinde, K.G. Chetyrkin, and S.A. Larin, Phys. Lett. B272, 39 (1991).

[20] W. Janke and H. Kleinert (unpublished). 


\section{Figure Headings}

Fig. 1: Fourth-order cumulant $U_{L}$ vs $\beta$. The values of $U_{L}(\beta)$ were obtained by reweighting and optimized combining the results of our three simulations at different temperatures for each lattice size $L$. The simulations were performed at $\beta_{0}=0.6929$ (the critical coupling found by Peczak et al. [3]), and at the temperature locations of the maxima of the specific heat $C$ and the susceptibility $\chi^{\mathrm{c}}$, respectively.

Fig. 2: Variation of the pseudo transition temperatures $T_{\chi_{\max }^{\mathrm{c}}}(L)$ and $T_{\mathrm{C}_{\max }}(L)$ with $L^{-1 / \nu}$, where $\nu=0.704(6)$ is our FSS estimate (see text). The fits yield estimates of $\beta_{c}=0.6930(3)(Q=1.0)$ and $\beta_{c}=0.6925(9)$ $(Q=0.80)$, respectively. 